\title{
ASSESSMENT OF THE RELATIVE RISK OF AN AIRCRAFT ACCIDENT USING THE ANALYTIC HUERARCHY PROCESS
}

\author{
Deborah M. Osborne and David L. Ross \\ Embry-Riddle Aeronautical University \\ Department of Computing and Mathematics \\ Daytona Beach, FL, USA 32114-3900 \\ Osborned@db.erau.edu/rossd@cts.db.erau.edu
}

\begin{abstract}
This paper presents an application of an AHP-related technique for modeling the relative risk associated with a controlled-flight-into-terrain (CFIT) aircraft accident. The Flight Operations Risk Assessment System (FORAS) is a risk management tool that monitors and reduces the risks associated with individual flights. A technique for computing relative risk when limited data are available to model risk and the risks involve rare events associated with catastrophic consequences is developed and presented. The technique uses AHP to structure a hierarchy and a polynomial model for computing relative accident risk with input weights generated using pairwise comparisons.
\end{abstract}

\section{Introduction}

The Flight Operations Risk Assessment System (FORAS) is a risk management tool that monitors and reduces the risks associated with individual flights. The project was initiated through the efforts of the Flight Safety Foundation, and development of the system is being conducted as a collaborative effort involving Embry-Riddle Aeronautical University (ERAU), the Natonal Center for Atmospheric Research (NCAR), and the Naval Research Laboratories (NRL). Ultimately, the system will be designed as a decision support tool for safety managers.

Many efforts have been made to identify risk factors that are present in aircraft accidents and incidents, using post-mortem accident reviews. Collection and analysis of routine operational flight data have been limited to proprietary projects conducted within particular airlines. Safety experts have used checklists to identify risk factors associated with particular accident risk categories. However, these checklists have a limited ability to quantify risk and a more precise technique for risk assessment is needed.

The risk categories of Controlled Flight Into Terrain (CFIT) and Injury due to Turbulence were selected as the focus of the early modeling efforts. In this paper, we focus on the CFIT model. The Turbulence model is developed in a similar manner. CFIT is defined as the contact of an airworthy aircraft with terrain or an obstacle, without prior awareness of the crew. Any attempt to model CFIT risk must address the complex elements involved in flight operations that are not easily quantified. Communications critical to mission success are continually taking place between crew members, between the crew and dispatch, and between the crew and air traffic control. The effectiveness of these communications is influenced by the experience of the individuals involved, fatigue factors, the flight environment, and procedures dictated by the regulatory agency or the airline. The contribution of many diverse factors to events taking place during arrival or departure makes probability estimates difficult to obtain. Similar difficulties arise when determining risk in other risk categories (e.g. injury due to turbulence, loss of control, runway incursion) and this has hindered the quantitative assessment of risk within the aviation industry.

CFIT accidents have been extensively analyzed, and the event sequences leading to many accidents have been carefully reconstructed. The checklist categorizing CFIT risk factors and risk reduction elements has received recognition within the airline industry as a useful tool for identifying contributions to CFIT risk. 
However, the quantitative assessment of CFIT risk based on this checklist lacks rigor, and the need for a more meaningful numerical assessment has been recognized.

The Analytic Hierarchy Process was used to structure and prioritize the diverse factors contributing to risk in the flight environment. Since extensive input from experts is required to effectively identify factors contributing to CFIT risk, the hierarchical structure employed in the preliminary FORAS model proved effective in facilitating communications between modelers and industry experts. As the model was developed, it became clear that the traditional AHP hierarchical structure was useful for generating coefficients in a simple polynomial that would provide a relative measure of CFIT risk associated with a specific flight. However, it was necessary to construct the hierarchy with inputs for specific flights as entries at the lowest level. This differs from the usual AHP application where the lowest level consists of distinct alternatives to be evaluated. In this paper, we use the essential elements of AHP with these flightspecific inputs to develop a polynomial model assessing the risk of an accident.

\section{The Hierarchy and a Relative Risk Polynomial}

Risk can be defined as the potential for realization of adverse consequences. In most cases, CFIT accidents result in multiple loss of life. The combination of the catastrophic nature of a CFIT accident and its relative rarity produce special challenges to the development of a usable risk model. In particular, assessment of risk associated with catastrophic events is not naturally modeled by a likelihood approach, where the probability of an accident is multiplied by a measure of the severity of the consequences. Such an approach can yield the same measure for a high probability event with mild consequences as it does for a low probability event with catastrophic consequences. Most people, however, would not view these scenarios as equivalent, even though they could produce similar numerical assessments in a likelihood context.

To develop a hierarchy-described CFIT risk, a diverse group of aviation professionals were consulted. This group included pilots, safety managers, air traffic controllers, and representatives from many professional organizations within the industry. The hierarchy obtained is shown in figures 1-4.

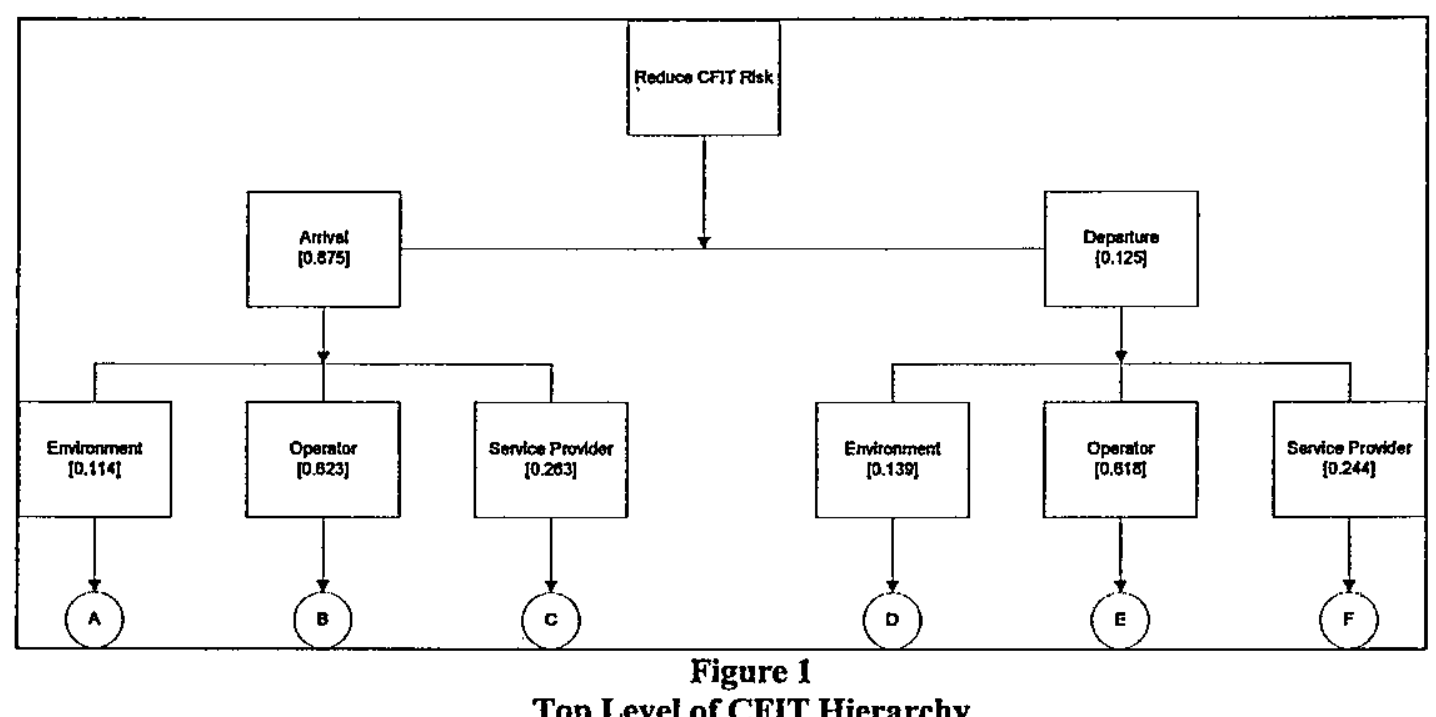

Figure 1 illustrates the top level distinction of Arrival and Departure below the goal of Identify CFIT Risk. The second level distinction of Environment, Operator, and Service Provider was agreed upon as a natural categorization of high level risk attributes. Environment includes both the physical and nonphysical attributes of the flight environment (e.g. navigation equipment as well as weather conditions). Operator refers to all aspects of the flight specific to the airline operator (e.g. crew, dispatch operations, 
and management issues). Service Provider refers to Air Traffic Control and accompanying issues pertaining to regulatory agencies.

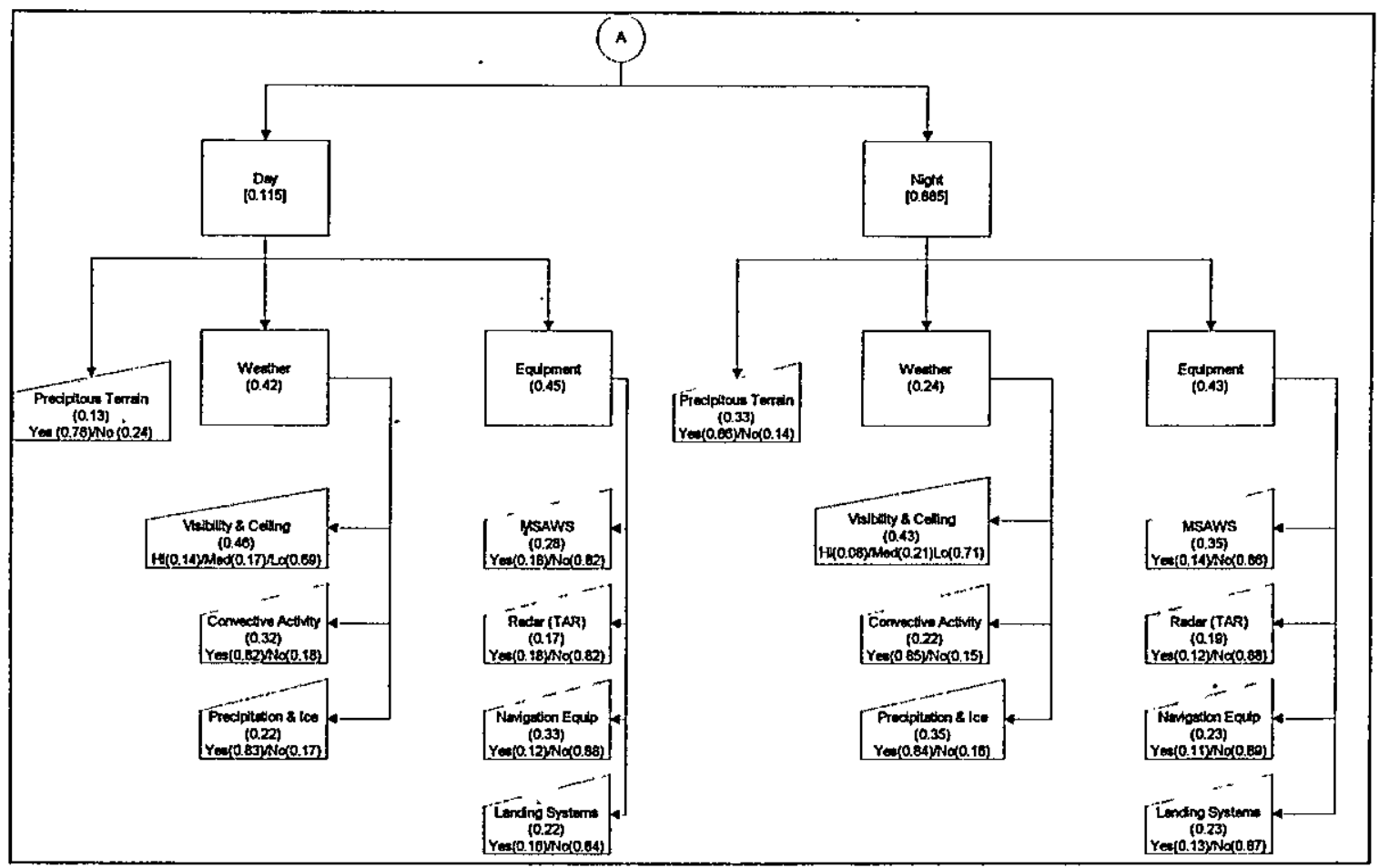

Figure 2

Environment Subhierarchy

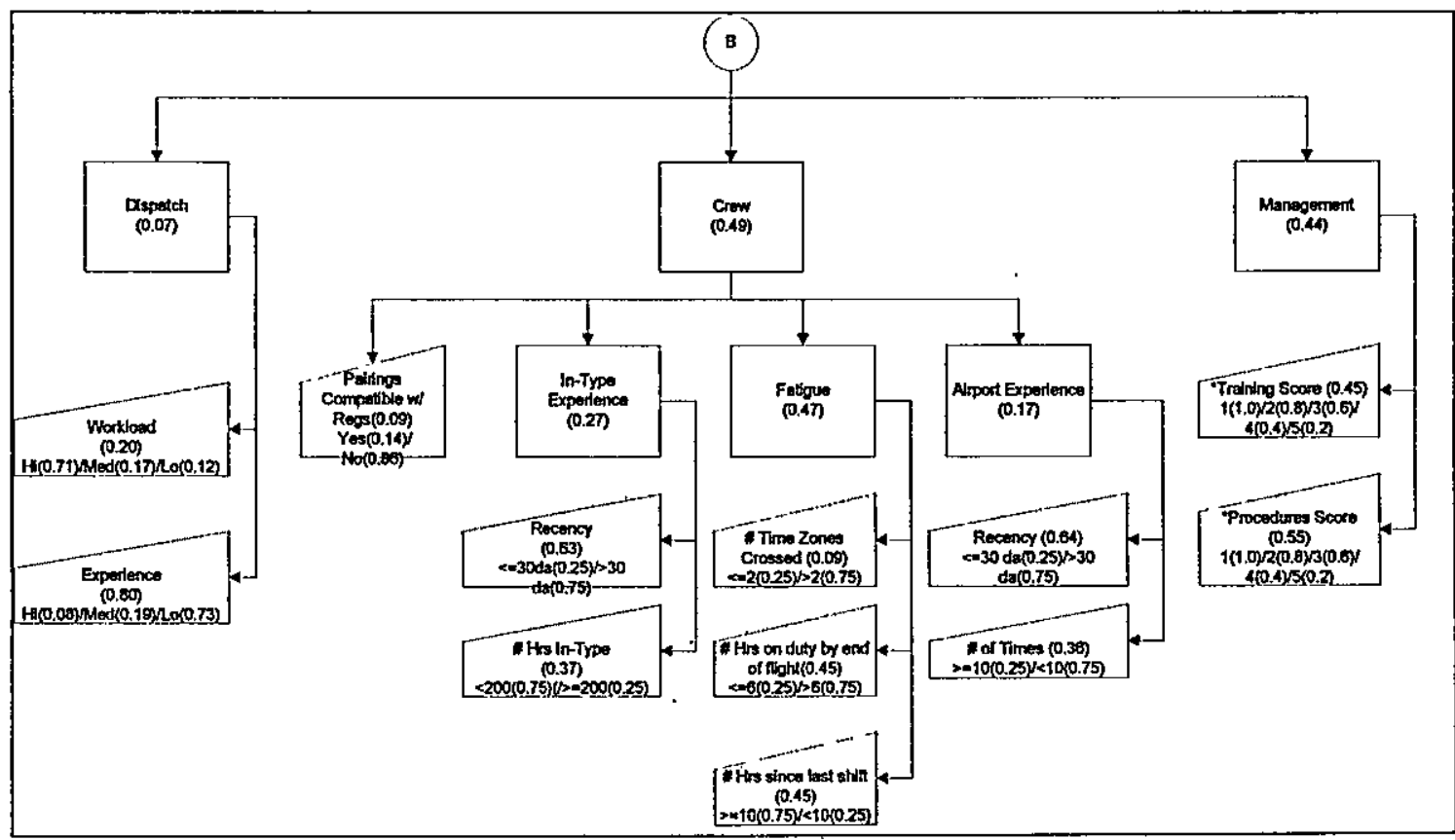

Figure 3

Operator Subhierarchy 
In this first phase, attention was focused on the arrival branch of the hierarchy. Subhierarchies (Figures 2-4) show the levels of categorization within Environment, Operator, and Service Provider, respectively. Pairwise comparisons were used to generate normalized eigenvectors describing the relative importance of attributes at each level of the hierarchy. The geometric mean of the preference ratios was used to synthesize the group judgments.

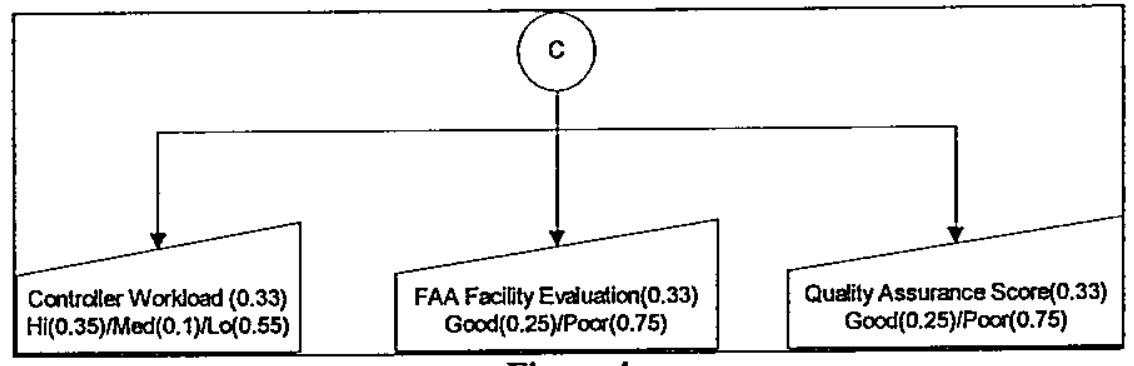

Figure 4

Service Provider Subhierarchy

At the lowest level of the hierarchy, indicated by trapezoids in figures $2-4$, inputs associated with an individual flight are indicated. These are not "alternatives" facing a decision-maker, as often occurs in multi-criteria decision models involving AHP. Rather, these inputs will be assigned a value, or input level, which reflects the state of the input for the flight under consideration. In the hierarchy, we distinguish between the goal, attribute node, input node, and input levels. For example, below the goal Identify CFIT Risk, the attribute nodes Arrival, Operator, and Dispatcher lead to the input nodes dispatch experience and dispatch workload. Each input node represents a measurable quantity associated with an individual flight. For example, both dispatch experience and dispatch workload are characterized by the input levels of high, medium, and low.

In the development of the model, expert input was used to provide priority weights on the input levels in the same way that priority weights were obtained for the attribute and input nodes. For example, an expert may be asked the following question: In the context of arrival, what constitutes a greater contribution to CFIT risk, a high level of dispatch experience or a medium level of dispatch experience? With the usual response as medium level, the expert is then asked: When considering contribution to CFIT risk, in the context of arrival, how much greater is the contribution of a medium level of dispatch experience compared to a high level of dispatch experience? A linguistic representation of a 1 to 9 scale was used to generate entries in the reciprocal matrix of preference ratios, and by completing the set of all such comparisons, priority vectors were generated for the input levels. Larger weights correspond to a greater CFIT risk and, for an individual flight, only one input level will be active for each input node. We will consider the weights on the active input levels to construct a relative risk polynomial that will assess CFIT risk.

To assess the risk of a particular flight, consider a collection of variables $x_{1}, x_{2} \ldots, x_{n}$ corresponding to the $n$ input nodes. Table 1 shows a pair of flight scenarios with assigned input levels.

Factor Abbreviations

DAYIPRECIPITOUS TERRAIN

DAYNEATHERNIS \& CEILING

DAYMEATHER/CONVECTIVE

DAYMEATHER/PRECIPIT \& ICE

DAYIEQUIPMENT/MSAWS

DAYIEQUIPMENT/RADAR

DAYIEQUIPMENT/NAVIG EQUIP

DAY/EQUIPMENT/LANDING SYS

NIGHT/PRECIPITOUS TERRAIN

NIGHTMEATHERNIS \& CEILING

\begin{tabular}{rrr} 
Risk Weights & Scenario A & \multicolumn{2}{c}{ Scenario B } \\
0.0017 & 0 & 0.24 \\
0.00253 & 0 & 0.14 \\
0.00176 & 0 & 0.18 \\
0.00121 & 0 & 0.17 \\
0.00165 & 0 & 0.18 \\
0.001 & 0 & 0.18 \\
0.00195 & 0 & 0.12 \\
0.0013 & 0 & 0.16 \\
0.03329 & 0.14 & 0 \\
0.01041 & 0.71 & 0
\end{tabular}




$\begin{array}{lrrr}\text { NIGHTMEATHER/CONVECTIVE } & 0.00533 & 0.85 & 0 \\ \text { NIGHT/WEATHER/PRECIPIT\&ICE } & 0.00847 & 0.84 & 0 \\ \text { NIGHT/EQUIPMENT/MSAWS } & 0.01518 & 0.14 & 0 \\ \text { NIGHT/EQUIPMENT/RADAR } & 0.00824 & 0.12 & 0 \\ \text { NIGHT/EQUIPMENT/NAV EQUIP } & 0.00998 & 0.11 & 0 \\ \text { NIGHT/EQUIPMENT/LAND SYS } & 0.00998 & 0.13 & 0 \\ \text { DISPATCHINORKLOAD } & 0.00872 & 0.12 & 0.12 \\ \text { DISPATCH/EXPERIENCE } & 0.03489 & 0.08 & 0.08 \\ \text { CREW/COMPATIBLE PAIRINGS } & 0.02747 & 0.14 & 0.14 \\ \text { CREW/N-TYPE EXP/RECENCY } & 0.05193 & 0.25 & 0.25 \\ \text { CREW/N-TYPE EXP/HOURS } & 0.0305 & 0.25 & 0.25 \\ \text { CREW/FATIGUE/TIME ZONES } & 0.01291 & 0.25 & 0.25 \\ \text { CREW/FATIGUE/HRS ON DUTY } & 0.06456 & 0.25 & 0.25 \\ \text { CREW/FATIGUE/HRS REST } & 0.06456 & 0.25 & 0.25 \\ \text { CREWIAIRPORT EXP/RECENCY } & 0.03321 & 0.25 & 0.25 \\ \text { CREWIAIRPORT/\# OF TIMES } & 0.01868 & 0.25 & 0.25 \\ \text { MANAGEMENT/TRAINING } & 0.12335 & 0.07 & 0.33 \\ \text { MANAGEMENT/PROCEDURES } & 0.15077 & 0.07 & 0.33 \\ \text { CONTROLLER WORKLOAD } & 0.08679 & 0.1 & 0.1 \\ \text { FAA FACILITY EVALUATION } & 0.08679 & 0.25 & 0.25 \\ \text { SERV PROVIDER/QA PROGRAM } & 0.08679 & 0.25 & 0.25 \\ \text { TOTAL RISK VALUE FOR INDIVIDUAL SCENARIOS* } & & 0.17724 & 0.221508\end{array}$

In the first flight scenario, the flight is flown in the most desirable circumstances, except the flight occurs at night in bad weather (Scenario A). In the second flight, the flight is flown in the most desirable circumstances, except the airline has poor management procedures and training (Scenario B). If $x_{i}$ denotes the priority weight for the $i^{\text {th }}$ input level, then the relative CFIT risk associated with the flight may be represented as a polynomial

$$
R\left(x_{1}, x_{2}, \ldots, x_{n}\right)=\sum_{i=1}^{n} \prod_{j=1}^{m} c_{i j} x_{i},
$$

where the coefficients $c_{i j}$ are given by the priority weights of the input node and attribute nodes on a path from the input level to the goal. For example, if $x_{1}$ represents dispatch experience, then in scenario A, $x_{1}=0.08$, and the coefficient of the $x_{1}$ term in (1) is given by 0.0349 .

By summing over all input nodes, an assessment of CFIT risk of 0.18 for scenario $A$ and 0.22 for scenario $\mathrm{B}$ is obtained. Larger values of $R$ will be obtained for flights that have larger input levels (corresponding to greater CFIT risk), and this effect will be magnified when the input level lies below an attribute node that is heavily weighted. While $R$ will range between a minimum of 0.15 and a maximum of 0.62 , no attempt is made to assign an absolute meaning to this value. Rather, the value of $R$ obtained for a specific flight reflects a relative measure of risk.

As data becomes available for a large collection of flights, the sensitivity of $R$ to the inputs and attribute weights may be used along with expert evaluation of CFIT risk to mathematically transform the relative risk scale in a meaningful way. Narrative descriptions of various levels of risk will be useful, especially when considering aggregate risk measures for collections of flights associated with a given route, a given airport, or a given flight environment.

Preliminary model verification was conducted by asking recognized industry experts to rank a collection of five flight scenarios from perceived highest CFIT risk to lowest CFIT risk. Kendall's W test showed that the experts were consistent in their rankings. These scenarios were then evaluated by the model, and a ranking established using the relative risk polynomial. The Wilcoxon signed rank test showed that there was no significant difference between the rankings provided by the experts and the rankings obtained by the model. While such results are valuable in demonstrating proof of concept, it is necessary to obtain input levels for a large number of actual flights to determine the validity of the model. These efforts are currently underway. 


\section{Conclusion and Future Phases}

This technique adds to the arsenal of probabilistic risk assessment techniques. It is especially useful for those cases where limited data are available to model risk and the risks involve rare events associated with catastrophic consequences. AHP is especially useful in structuring the hierarchy and the polynomial model with input weights derived using pairwise comparisons provides a valuable tool for modeling relative risk.

Phase 2 work will be completed early in 2000. A prototype expert system assessing CFIT risk at United Airlines will be tested by the end of Phase 2. During Phase 2, input nodes will be refined to enable continuous variables to be represented, dependencies between branches of the hierarchy will be modeled, and user requirements will be ascertained. Analysis and testing will be performed using real flight routes. Climatological databases, as well as databases with weather and forecast information, will be developed for these routes. Finally, real airline data will be used for the model development, analysis, and testing.

We would like to acknowledge the contribution of our colleagues at NCAR: Barbara Brown, Diana Boyd, and Bill Mahoney, and our colleague at NRL: Michael Hadjimichael. Additionally, our gratitude extends to the two people who have championed this project from its infancy: John McCarthy and Douglas Schwartz.

\section{Bibliography}

Khatwa, R. and Roelen, A.L.C. (1996) An Analysis of CFIT Accidents of Commercial Operators, 1988 Through 1994, Flight Safety Digest, April-May 1996.

Lehmann, E.L. (1975) Nonparametrics: Statistical Methods Based on Ranks, Holder-Day, Inc., Oakland, CA.

Roelen, A.L.C. and van de Nat, G.W.F.M. (1998) The Development of Aviation Safety Performance Indicators: An Exploratory Study. NLR-CR-98183. Netherlands National Aerospace Laboratory NLR, Amsterdam.

Saaty, Thomas L. (1994) Fundamentals of Decision Making and Priority Theory, RWS Publications, Pittsburgh, PA. 